\title{
Structural and electrolyte properties of $\mathrm{Li}_{4} \mathrm{P}_{2} \mathrm{~S}_{6}$
}

\author{
Zachary D. Hood ${ }^{\mathrm{a}, 1}$, Cameron Kates ${ }^{\mathrm{b}, 2}$, Melanie Kirkham ${ }^{\mathrm{c}}$, Shiba Adhikari ${ }^{\mathrm{d}}$, Chengdu Liang, ${ }^{\mathrm{a}, 3}$, N. A. W. Holzwarth ${ }^{\mathrm{b}, 4}$ \\ ${ }^{a}$ Center for Nanophase Materials Sciences, Oak Ridge National Laboratory, Oak Ridge, TN 37831 USA \\ ${ }^{b}$ Department of Physics, Wake Forest University, Winston-Salem, NC 27109-7507 USA \\ ${ }^{c}$ Spallation Neutron Source, Oak Ridge National Laboratory, Oak Ridge, TN 37831 USA \\ ${ }^{d}$ Department of Chemistry, Wake Forest University, Winston-Salem, NC 27109-7486 USA
}

\begin{abstract}
Experiment and simulations are used to investigate the structural and electrolyte properties of $\mathrm{Li}_{4} \mathrm{P}_{2} \mathrm{~S}_{6}$. Compared with other thiophosphate materials, $\mathrm{Li}_{4} \mathrm{P}_{2} \mathrm{~S}_{6}$ is quite stable, maintaining its crystal structure up to temperatures as high as $950^{\circ} \mathrm{C}$ in vacuum and up to $280^{\circ} \mathrm{C}$ in air. While its ionic conductivity is small, $2.38 \times 10^{-7} \mathrm{~S} / \mathrm{cm}^{\circ}$ at $25^{\circ} \mathrm{C}$ and $2.33 \times 10^{-6} \mathrm{~S} / \mathrm{cm}$ at $100^{\circ} \mathrm{C}$, its Arrhenius activation energy of $0.29 \mathrm{eV}$ is similar to technologically viable electrolytes. Computer simulations provide insight into the causes and effects of disorder in this material and also indicate that the mechanism of the ion conduction is dominated by interstitial sites.
\end{abstract}

\section{Introduction}

Recently, there has been renewed interest in the development of all-solid state batteries, $[1,2,3]$ following the discovery of highly conducting solid electrolytes such as $\mathrm{Li}_{10} \mathrm{GeP}_{2} \mathrm{~S}_{12}[4]$ and nano-porous $\beta$ - $\mathrm{Li}_{3} \mathrm{PS}_{4}$. [5] Development of the all solid state battery technology motivates continued research on solid electrolytes focusing both on the continued improvement of ionic conductivity and on increasing the structural and chemical stability.

As an example of a relatively stable solid electrolyte, $\mathrm{Li}_{4} \mathrm{P}_{2} \mathrm{~S}_{6}$ has been identified in several high temperature preparations of lithium thiophosphate electrolytes as a synthesis or decomposition product.[6, 7, 8] Its characteristic $\mathrm{P}-\mathrm{P}$ bond may be partly responsible for its relative stability. Early structural analysis[9] found the $\mathrm{P}$ sites to be disordered. Our previous simulations[10] found a related low energy structure with ordered $\mathrm{P}$ sites. We report here a re-examination of the simulation results and new measurements which reveal interesting details of the structure and electrolyte properties of $\mathrm{Li}_{4} \mathrm{P}_{2} \mathrm{~S}_{6}$.

Section 2 details the experimental and simulation methods used in this study. Section 3.1 presents results on the synthesis and sample morphology. Structural properties are presented in Sec. 3.2 including a consideration of the effects of the disordered placement of the $\mathrm{P}_{2} \mathrm{~S}_{6}$ building blocks. Results concerning the stability of $\mathrm{Li}_{4} \mathrm{P}_{2} \mathrm{~S}_{6}$ are presented in Sec. 3.3. Electrochemical results and simulations are presented in Sec. 3.4. A summary and conclusions are given in Sec. 4.

\footnotetext{
${ }^{1}$ Current affliation: School of Chemistry and Biochemistry, Georgia Institute of Technology

${ }^{2}$ Current affliation: Pratt School of Engineering, Duke University.

${ }^{3}$ Current affiliation: Research Institute of Ningde Amperex Technology Limited

${ }^{4}$ Corresponding author - email:natalie@wfu.edu
}

\section{Methods}

\subsection{Experimental procedures}

\subsubsection{Synthesis of $\mathrm{Li}_{4} \mathrm{P}_{2} \mathrm{~S}_{6}$}

The synthesis of $\mathrm{Li}_{4} \mathrm{P}_{2} \mathrm{~S}_{6}$ was based on high temperature solid state reaction methods similar to procedures found in the literature.[9, 11] Reagent-grade $\mathrm{Li}_{2} \mathrm{~S}$ (Alfa Aesar, 99.9\%) and $\mathrm{P}_{2} \mathrm{~S}_{5}$ (Sigma-Aldrich, 99\%) crystalline powders were ground with a mortar and pestle for 20 minutes and sealed in an evacuated quartz tube to form lithium thiophosphate using the following reaction:

$$
2 \mathrm{Li}_{2} \mathrm{~S}+\mathrm{P}_{2} \mathrm{~S}_{5} \rightarrow \mathrm{Li}_{4} \mathrm{P}_{2} \mathrm{~S}_{6}+\mathrm{S} .
$$

Since the starting materials are hygroscopic and sensitive to air, this preparation was performed in an Ar-filled glove box.

Because we have found that the same phase of $\mathrm{Li}_{4} \mathrm{P}_{2} \mathrm{~S}_{6}$ can be formed at temperatures as low as $750^{\circ} \mathrm{C}$ and at temperatures as high as $900^{\circ} \mathrm{C}$, two synthetic routes were explored. For the higher temperature synthesis, the prepared powder was heated to $900^{\circ} \mathrm{C}$ for 20 hours and held at $450^{\circ} \mathrm{C}$ for 24 hours in an electric furnace with a ramp up/down of $100^{\circ} \mathrm{C} /$ hour. The reaction product contained elemental sulfur which was removed by stirring the powder in anhydrous acetronitrile for one hour. The powder was then filtered, and dried at $150^{\circ} \mathrm{C}$ under vacuum for 2 hours, producing a phase-pure material, as evidenced by $\mathrm{X}$-ray diffraction data. For the lower temperature synthesis, $\mathrm{Li}_{4} \mathrm{P}_{2} \mathrm{~S}_{6}$ was heated to $750^{\circ} \mathrm{C}$ for 48 hours with a ramp up/down at a rate of $100^{\circ} \mathrm{C} /$ hour, and after similar processing in anhydrous acetronitrile, the reaction yields the same phase-pure product. Both synthetic routes yielded $\mathrm{Li}_{4} \mathrm{P}_{2} \mathrm{~S}_{6}$ with the same electrolyte properties. 


\subsubsection{Temperature dependent structural characterization}

A PANalytical X'Pert Pro Powder Diffractometer with copper $K_{\alpha}$ radiation was used to complete all of the X-ray diffraction (XRD) analysis. Samples were placed on quartz slides for XRD analysis. Low temperature XRD measurements were completed using the Oxford Systems PheniX Cryostat over the range $15 \leq T \leq 298 \mathrm{~K}$. The software package HighScore Plus was used to complete Rietveld refinement and structural analysis. Scanning electron microscopy (SEM) images were collected using a Bruker Merlin SEM.

In addition, time-of-flight neutron diffraction results were collected at Oak Ridge National Laboratory using the POWGEN powder diffractometer at the Spallation Neutron Source. Samples were prepared with $700 \mathrm{mg}$ of $\mathrm{Li}_{4} \mathrm{P}_{2} \mathrm{~S}_{6}$ loaded into a vanadium can (diameter $6 \mathrm{~mm}$ ), sealed with an aluminum lid and copper gasket, and fitted with a titanium collar. The time-of-flight neutron diffraction patterns were collected at $300 \mathrm{~K}$ and $15 \mathrm{~K}$ over the range of diffraction plane $d$ spacings of $0.4-9.0 \AA$. The time-of-flight neutron refinements were completed using the GSAS and EXPGUI software packages.[12]

The thermal response of $\mathrm{Li}_{4} \mathrm{P}_{2} \mathrm{~S}_{6}$ was measured by using a TA Q600 differential scanning calorimeter fitted with a platinum/platinum-rhodium thermocouple. Measurements were taken on a $4.0 \mathrm{mg}$ sample of $\mathrm{Li}_{4} \mathrm{P}_{2} \mathrm{~S}_{6}$ placed into a ceramic cup and heated to $970^{\circ} \mathrm{C}$ at a rate of $10^{\circ}$ $\mathrm{C} / \mathrm{min}$ under conditions of either constant flow of air or constant flow of argon gas. The percent weight loss was calculated from the initial weight of the sample.

\subsubsection{Electrochemical Characterization}

In order to prepare the sample for electrochemical measurements, further processing was undertaken in the glove box. First, ball milling with $\mathrm{Y}-\mathrm{ZrO}_{2}$ beads having a mixture of 3 and $5 \mathrm{~mm}$ diameter using a ratio of $1: 25\left(\mathrm{Li}_{4} \mathrm{P}_{2} \mathrm{~S}_{4}\right.$ : ball milling media) in an 8000M Spex MixerMill was used to reduce the $\mathrm{Li}_{4} \mathrm{P}_{2} \mathrm{~S}_{6}$ particle size. Then, the powder was cold-pressed at $300 \mathrm{MPa}$ to form a pellet. XRD data confirmed that the ball milling media did not react with $\mathrm{Li}_{4} \mathrm{P}_{2} \mathrm{~S}_{6}$.

Ionic conductivity measurements were completed using a Solartron 1260 impedance spectrometer over the frequency range $1 \mathrm{~Hz}-1 \mathrm{MHz}$ with an amplitude of $100 \mathrm{mV}$. Arrhenius measurements used a Maccor Environmental chamber over the range $25^{\circ} \mathrm{C} \leq T \leq 100^{\circ} \mathrm{C}$. The samples were formed into pellets of diameter $1.27 \mathrm{~cm}$ and were pressed with $\mathrm{Al} / \mathrm{C}$ blocking electrodes at $300 \mathrm{MPa}$ in a pressurized cell for all electrochemical measurements.

\subsection{Simulation formalisms and algorithms}

The calculational methods used in this work are the same as that used in previous studies of similar materials.[13] Specifically, the calculations are based on density functional theory, $[14,15]$ using the projector augmented wave (PAW)[16] formalism. The PAW basis and projector functions were generated by the ATOMPAW[17] code and used in both the ABINIT[18] and QUANTUM ESPRESSO[19] packages. The exchange-correlation functional was the local density approximation (LDA),[20] which has been shown to give excellent results for similar materials provided that a systematic $2 \%$ underestimate of the lattice size is taken into account for each of the 3 dimensions. The simulated results reported here (including the simulations of the diffraction patterns) have all been adjusted by multiplying the calculated lengths by 1.02. The calculated fractional coordinates and total energies are assumed to be correct.

The electronic structure calculations were performed with plane wave expansions of the wavefunctions including $|\mathbf{k}+\mathbf{G}|^{2} \leq 64$ bohr $^{-2}$ and with a Brillouin zone sampling grid density of at least $0.003 \mathrm{bohr}^{-3}$. Structural parameters of the model systems were determined by minimizing the calculated total energies. The electronic structure results could be qualitatively analyzed in terms of partial densities of states $N^{a}(E)$, calculated from the expression

$$
N^{a}(E)=\sum_{n \mathbf{k}} W_{\mathbf{k}} Q_{n \mathbf{k}}^{a} \delta\left(E-E_{n \mathbf{k}}\right)
$$

as described in previous work.[13] Here $a$ denotes an atomic site, $W_{\mathbf{k}}$ denotes the Brillouin zone sampling weight factor for wave vector $\mathbf{k}$, and $E_{n \mathbf{k}}$ denotes the band energy for band index $n$ and wave vector $\mathbf{k}$. In practice, the $\delta$ function is represented by a Gaussian smoothing function with a width of $0.14 \mathrm{eV}$. For each eigenstate $n \mathbf{k}$ and atomic site $a$, the local density of states factor $Q_{n \mathbf{k}}^{a}$ is given by the charge within the augmentation sphere of radius $r_{c}^{a}$ which can be well approximated by

$$
Q_{n \mathbf{k}}^{a} \approx \sum_{i j}\left\langle\widetilde{\Psi}_{n \mathbf{k}} \mid p_{n_{i} l_{i} m_{i}}^{a}\right\rangle\left\langle p_{n_{j} l_{i} m_{i}}^{a} \mid \widetilde{\Psi}_{n \mathbf{k}}\right\rangle q_{n_{i} l_{i} ; n_{j} l_{i}}^{a} \delta_{l_{i} l_{j}}
$$

in terms of the radial integrals

$$
q_{n_{i} l_{i} ; n_{j} l_{i}}^{a} \equiv \int_{0}^{r_{c}^{a}} d r \varphi_{n_{i} l_{i}}^{a}(r) \varphi_{n_{j} l_{j}}^{a}(r) .
$$

In these expressions, $\left|\widetilde{\Psi}_{n \mathbf{k}}\right\rangle$ represents a pseudo-wavefunction, $\left|\widetilde{p}_{n_{i} l_{i} m_{i}}^{a}\right\rangle$ represents a PAW atomic projector function localized within the augmentation sphere about atomic site $a$ and characterized with radial and spherical harmonic indices $n_{i} l_{i} m_{i}$. $[16,21]$ The function $\varphi_{n_{i} l_{i}}^{a}(r)$ represents the corresponding all-electron radial basis function. The augmentation radii used in this work are $r_{c}^{\mathrm{Li}}=1.6, r_{c}^{\mathrm{O}}=1.2$, $r_{c}^{\mathrm{P}}=1.7$, and $r_{c}^{\mathrm{S}}=1.7 \mathrm{in}$ bohr units. The reported partial densities of states, $\left\langle N^{a}(E)\right\rangle$, are averaged over sites of each type $a$.

The "nudged elastic band" (NEB) method, [22, 23, 24] as programmed in the QUANTUM ESPRESSO package was used to estimate the $\mathrm{Li}^{+}$migration energies $E_{m}$ in supercell models. For this analysis it was assumed that 5 images between each meta-stable configuration was sufficient to estimate the path energies. For these simulations, supercells consisting of 8 formula units were used. The simulations were performed on neutral supercells, either 
by using a compensating uniform charge, or by modeling an interstitial ion in the presence of a distant vacancy (or visa versa) within the supercell. The migration energies determined from the NEB calculations can be related to the experimental conductivity $\sigma$ measurements as a function of temperature $T$ through the Arrhenius relationship ${ }^{5}$

$$
\sigma=\sigma_{0} \mathrm{e}^{-E_{A} / k T},
$$

where $k$ denotes the Boltzmann constant, $E_{A}$ represents the activation energy for $\mathrm{Li}$ ion migration, and $\sigma_{0}$ denotes a temperature independent constant for the sample. For nearly perfect crystals, thermal processes must initiate the formation of a vacancy and interstitial pair with energy $E_{f}$ so that $E_{A}=E_{m}+\frac{1}{2} E_{f}$. For crystals with a significant population of native defects, we expect $E_{A}=E_{m}$.

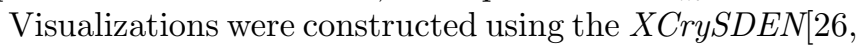
27] and VESTA[28] software packages. The software program FINDSYM[29] was used to help analyze the symmetry properties of the optimized structures. In order to directly compare the model structures with the diffraction measurements, powder patterns were generated from the calculated structural parameters using the program Mercury[30] for the X-ray patterns and using the program GSAS[12] for the neutron patterns. The optimized fractional coordinates were used directly, while, as mentioned above, all of the calculated lattice parameters were scaled by a uniform factor of 1.02 in order correct for the systematic size error of the local density approximation (LDA) exchange correlation functional. The diffraction results of Mercier et al.[9] reported here were also generated using the Mercury and GSAS programs.

\section{Results}

\subsection{Crystal Properties}

$\mathrm{Li}_{4} \mathrm{P}_{2} \mathrm{~S}_{6}$ was found to form at increased temperatures between $750^{\circ} \mathrm{C}$ and $900^{\circ} \mathrm{C}$ in vacuum. The yield of each synthesis process described in Sec. 2.1.1 was $99 \%$. The purified reaction product included particles having diameters $\geq 10 \mu \mathrm{m}$ which were reduced to nanosize after ball-milling. SEM images of the sample after the ball-milling process at two different magnifications are shown in Fig. 1a and 1b. Producing the nanosized powder aided in pressing dense pellets for electrochemical characterization. This method resulted in pellets as dense as $96 \%$ of the ideal density of $2.23 \mathrm{~g} / \mathrm{cm}^{3}$.

\subsection{Structural Properties}

As noted in 1982 by Mercier et al., [9] the building blocks of $\mathrm{Li}_{4} \mathrm{P}_{2} \mathrm{~S}_{6}$ crystals are $\mathrm{P}_{2} \mathrm{~S}_{6}$ (hexathiohypodiphosphate) ions having $\mathrm{D}_{3 d}$ symmetry, oriented with the $\mathrm{P}-\mathrm{P}$

\footnotetext{
${ }^{5}$ This form of the Arrhenius relation lacks the $1 / T$ prefactor which comes from the Einstein relation between diffusion and ionic conductivity, such as derived in Ref. [25]. However, this form empirically fits the data well.
}
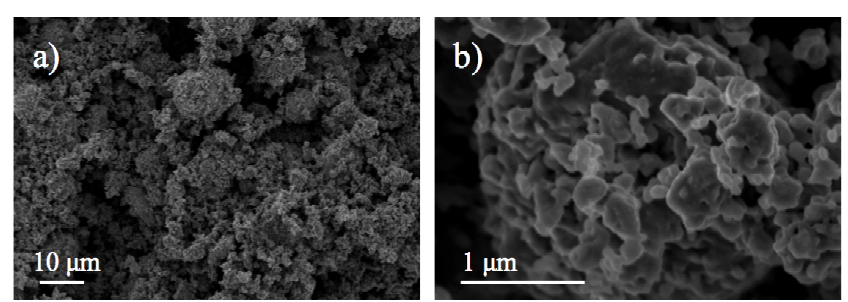

Figure 1: Scanning electron microscopy (SEM) images of $\mathrm{Li}_{4} \mathrm{P}_{2} \mathrm{~S}_{6}$ (a) after ball milling, and (b) a close-up of the ball milled material showing nanosized particles.

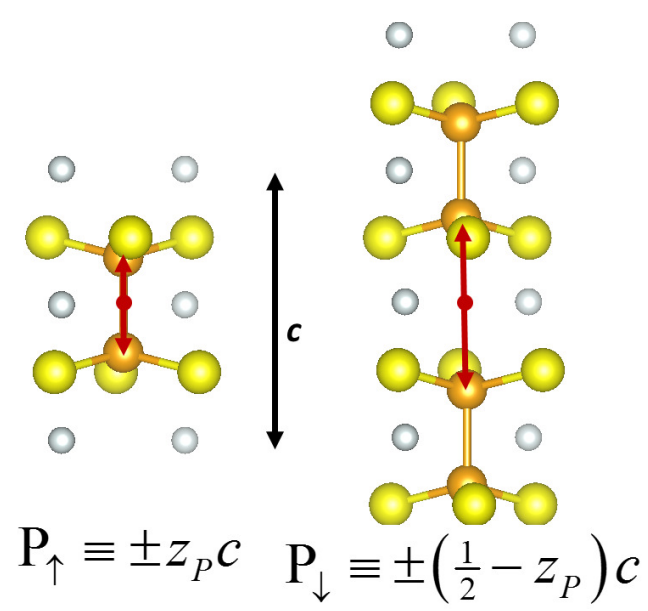

Figure 2: (Color online.) Ball-and-stick diagram of the $\mathrm{P}_{2} \mathrm{~S}_{6}$ ion units comprising the $\mathrm{Li}_{4} \mathrm{P}_{2} \mathrm{~S}_{6}$ crystals. The ball designations are $\mathrm{Li}$, $\mathrm{P}, \mathrm{S}$ in increasing size with gray, orange, and yellow colors respectively. The red arrows indicate the two possible placements of the $\mathrm{P}$ sites within a unit cell centered at the red dot.

bond along the crystallographic $c$ axis, as shown in Fig. 2. The left diagram of the figure shows one possible placement of a $\mathrm{P}_{2} \mathrm{~S}_{6}$ ion with the origin of the unit cell at the center of the $\mathrm{P}-\mathrm{P}$ bond and the coordinates of the $\mathrm{P}$ sites designated as $\mathrm{P}_{\uparrow} \equiv \pm z_{P} c$. The right diagram of the figure shows the other possible placement of the building blocks with the origin of the unit cell located between $\mathrm{P}_{2} \mathrm{~S}_{6}$ units and the coordinates of the $\mathrm{P}$ sites designated as $\mathrm{P}_{\downarrow} \equiv \pm\left(\frac{1}{2}-z_{P}\right) c$.

Mercier et al.[9] described the overall structure of $\mathrm{Li}_{4} \mathrm{P}_{2} \mathrm{~S}_{6}$ in terms of the space group $P 6_{3} / \mathrm{mcm}$ (\# 193). [31] In this designation, using the site multiplicity and Wyckoff symbols, the $4 e \mathrm{P}$ sites at fractional coordinates $\left(0,0, z_{P}\right)$, and three other symmetry equivalent sites, have 0.5 occupancy. On the other hand, the $4 d \mathrm{Li}$ sites at fractional coordinates $(1 / 3,2 / 3,0)$, and three other symmetry equivalent sites, and the $6 g \mathrm{~S}$ sites at fractional coordinates $\left(x_{S}\right.$, $0,1 / 4)$, and five other symmetry equivalent sites, are fully occupied.

The basic structure of the crystal as projected within a hexagonal plane is shown in Fig. 3a. This structure is common to all of the possible structural variations. The placement of any given $\mathrm{P}-\mathrm{P}$ bond within a crystal unit determines the placement of all of the $\mathrm{P}-\mathrm{P}$ bonds along 
(a)

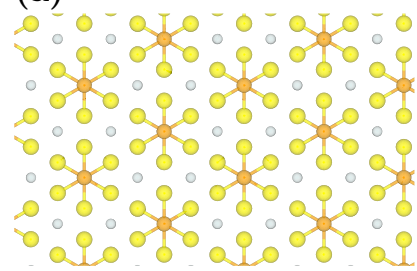

(c)

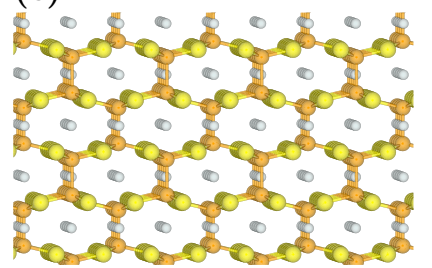

(b)

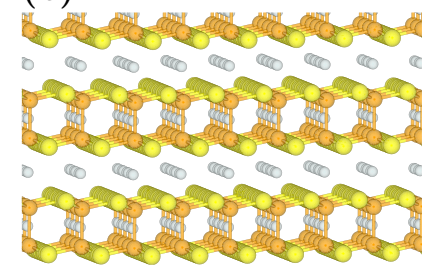

(d)

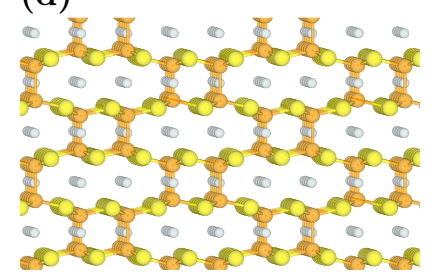

Figure 3: (Color online.) Ball-and-stick models of $\mathrm{Li}_{4} \mathrm{P}_{2} \mathrm{~S}_{6}$ with gray, orange, and yellow balls representing $\mathrm{Li}, \mathrm{P}$, and $\mathrm{S}$ sites, respectively. (a) Shows a projection onto the hexagonal plane common to all of the structures. Parts (b)-(d) show a view point with perpendicular and parallel components of the hexagonal plane. (b) Shows the ordered $P \overline{3} 1 \mathrm{~m}$ structure with an energy/formula unit of $0.03 \mathrm{eV}$ higher than the ground state. (c) Shows an ordered ground state configuration having two formula units per unit cell and Pnnm symmetry. (d) Shows an ordered ground state configuration having four formula units per unit cell and Pnma symmetry.

the same $c$ axis. It is reasonable to assume, as is consistent with the experimental analysis, that the $\mathrm{S}$ and Li sites are insensitive to these bond placements. The likely structural variations in $\mathrm{Li}_{4} \mathrm{P}_{2} \mathrm{~S}_{6}$ crystals can be enumerated on a two-dimensional hexagonal lattice in terms of the $P_{\uparrow}$ or $P_{\downarrow}$ placements of $\mathrm{P}-\mathrm{P}$ bonds on each $c$ axis. Our previous simulation studies[10] found a meta-stable structure which corresponds to choosing all of $\mathrm{P}-\mathrm{P}$ bonds of the $P_{\uparrow}$ type which results in a structure with group symmetry of $P \overline{3} 1 \mathrm{~m}$ (\# 162),[31] which is a subgroup of the Mercier structure. This structure of ordered $\mathrm{P}-\mathrm{P}$ bonds has an energy of $0.03 \mathrm{eV} /$ formula unit higher than the lowest energy structure, and is shown in Fig. 3b. Other possible structures have $\mathrm{P}-\mathrm{P}$ bonds in between alternate $\mathrm{S}$ layers. For example, the ordered structures shown in Figs. 3c and $3 \mathrm{~d}$ are based on orthorhombic supercells of the hexagonal structure having an equal number of $P_{\uparrow}$ and $P_{\downarrow}$ sites. These are two examples of structures computed to have the lowest energy of the configurations considered. The configuration of Struc. (c) has two formula units per unit cell and the the crystallographic space group is Pnnm (\# 58).[31] The configuration of Struc. (d) has four formula units per unit cell and the crystallographic space group is Pnma ( \# 62).[31] In addition to Struc. (c) and Struc. (d), we have examined several other configurations, generally finding that those configurations with equal numbers of $P_{\uparrow}$ and $P_{\downarrow}$ sites have energies equal to that of the ground state, while configurations with unequal numbers of $P_{\uparrow}$ and $P_{\downarrow}$ sites have energies between that of the ground state and $0.03 \mathrm{eV}$ which characterizes the pure $P_{\uparrow}$ setting. While we have by no means explored the vast configuration space

of this system, we can reasonably conclude that this system has many configurations corresponding to the ground state and many configurations with energies close to that of the ground state.

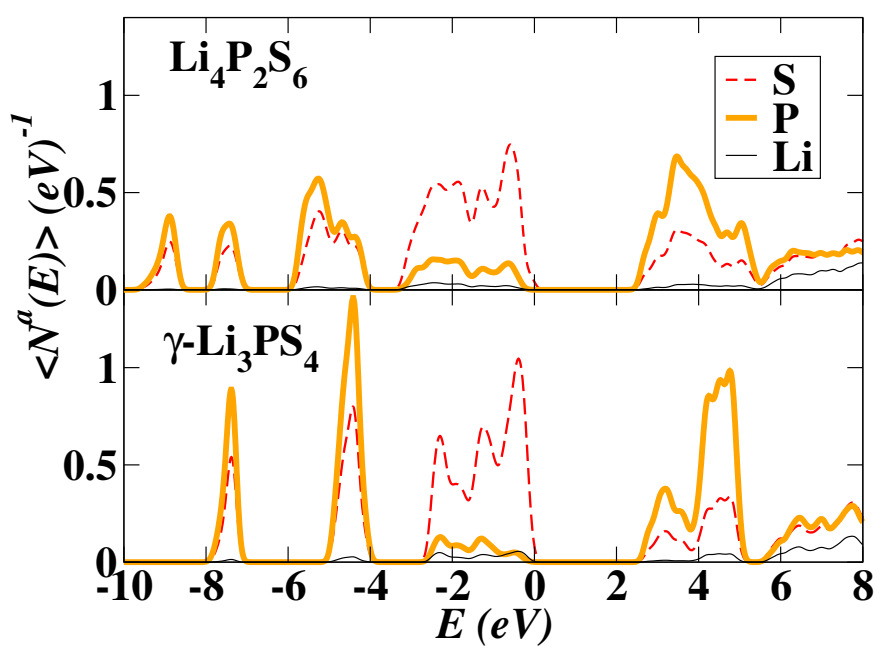

Figure 4: (Color online.) Partial density of states evaluated using Eq. 2 for $\mathrm{Li}_{4} \mathrm{P}_{2} \mathrm{~S}_{6}$ compared with that of $\gamma-\mathrm{Li}_{3} \mathrm{PS}_{4}$. The zero of energy is taken as the highest occupied state of the systems.

In addition to the structural optimization, the computer simulations determine the electronic structure of the materials, the qualitative features of which are exhibited by the partial densities of states. We find the partial densities of states of $\mathrm{Li}_{4} \mathrm{P}_{2} \mathrm{~S}_{6}$ to be insensitive to the detailed structures shown in Fig. 3. Figure 4 shows the partial densities of states for Struc. (d) shown in Fig. 3d and is very similar to the result for the $P \overline{3} 1 m$ structure.[10] The partial densities of states show that the $\mathrm{P}-\mathrm{P}$ dimers result in valence band contributions at $1.5 \mathrm{eV}$ lower energy and generally increase the overall valence band width compared to that of $\gamma-\mathrm{Li}_{3} \mathrm{PS}_{4}$.

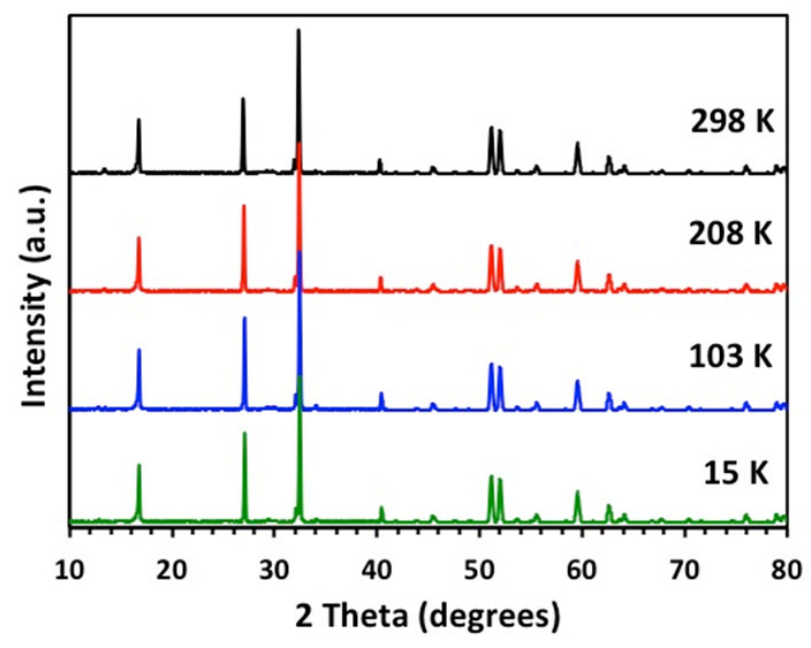

Figure 5: (Color online.) X-ray powder patterns of $\mathrm{Li}_{4} \mathrm{P}_{2} \mathrm{~S}_{6}$ measured at indicated temperatures. 


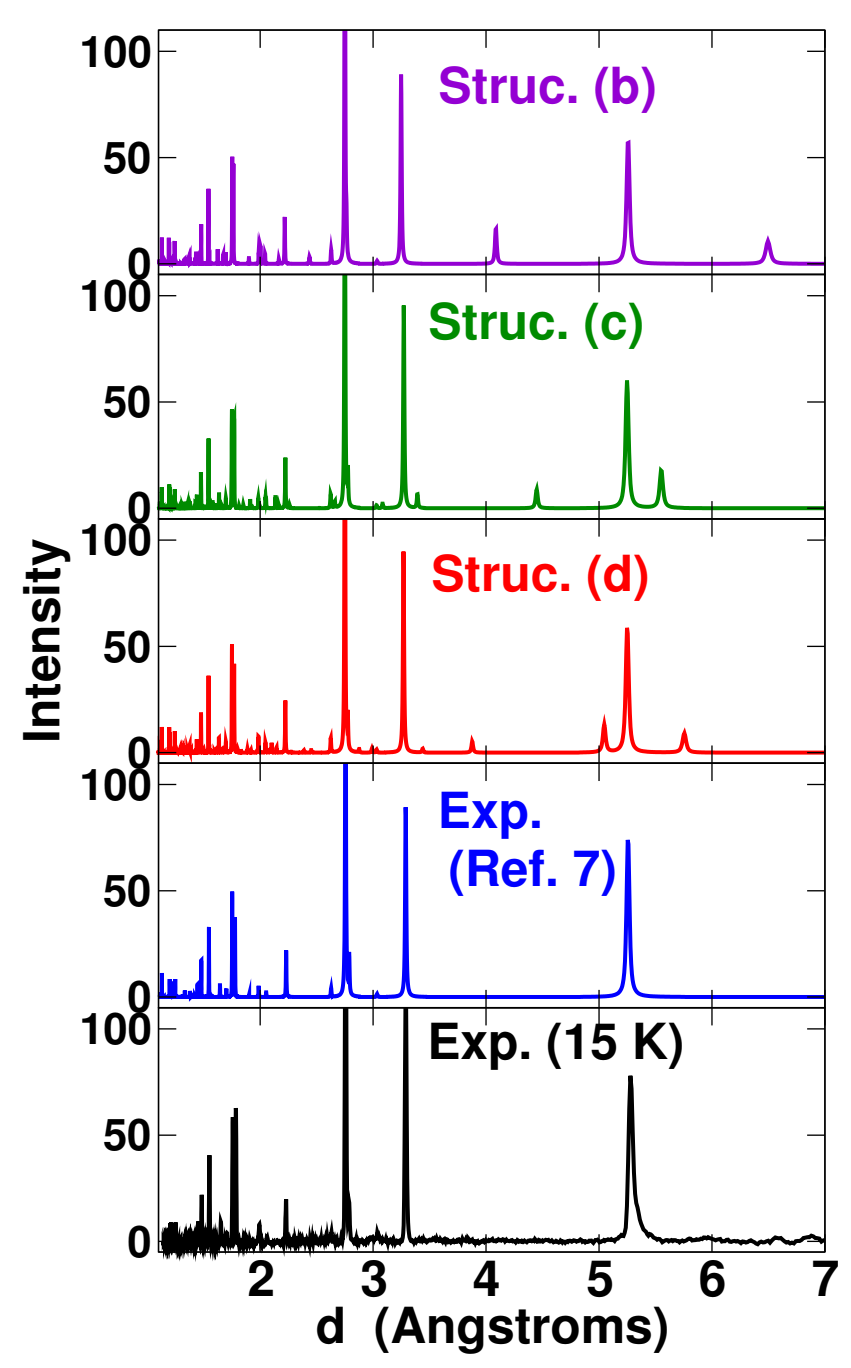

Figure 6: (Color online.) Simulated X-ray patterns from the structural models shown in Fig. 3, compared with our low temperature Xray data and the room temperature X-ray data reported by Mercier et al.[9] The intensity of the data and simulations were normalized so that the highest peaks at approximately $d=2.75 \AA$ are fixed at the intensity of approximately 200.

X-ray analysis of the powdered sample was carried out at room temperature and at several lower temperatures and the results are shown in Fig. 5. We expected $P_{\uparrow}$ and $P_{\downarrow}$ variations in the structures to result in a temperature dependence of the X-ray data. However, the fact that the 4 patterns are essentially identical indicates no structural transformation in the temperature range $15 \mathrm{~K} \leq T \leq 298 \mathrm{~K}$. This result is somewhat surprising given that calculations have identified many configurations of the $\mathrm{P}-\mathrm{P}$ bonds resulting in energies in the range of $0 \leq E \leq 0.03 \mathrm{eV} /$ formula units relative to the ground state. On the other hand, one expects that there may be significant energy barriers to changing $\mathrm{P}-\mathrm{P}$ bond configurations, involving either migrations of $\mathrm{P}_{2} \mathrm{~S}_{6}$ units or sequences of breaking and reforming $\mathrm{P}-\mathrm{P}$ bonds. This reasoning leads to the suggestion that the $\mathrm{P}-\mathrm{P}$ bond configurations might be "frozen" in at the time that the crystal is formed. The room tem-

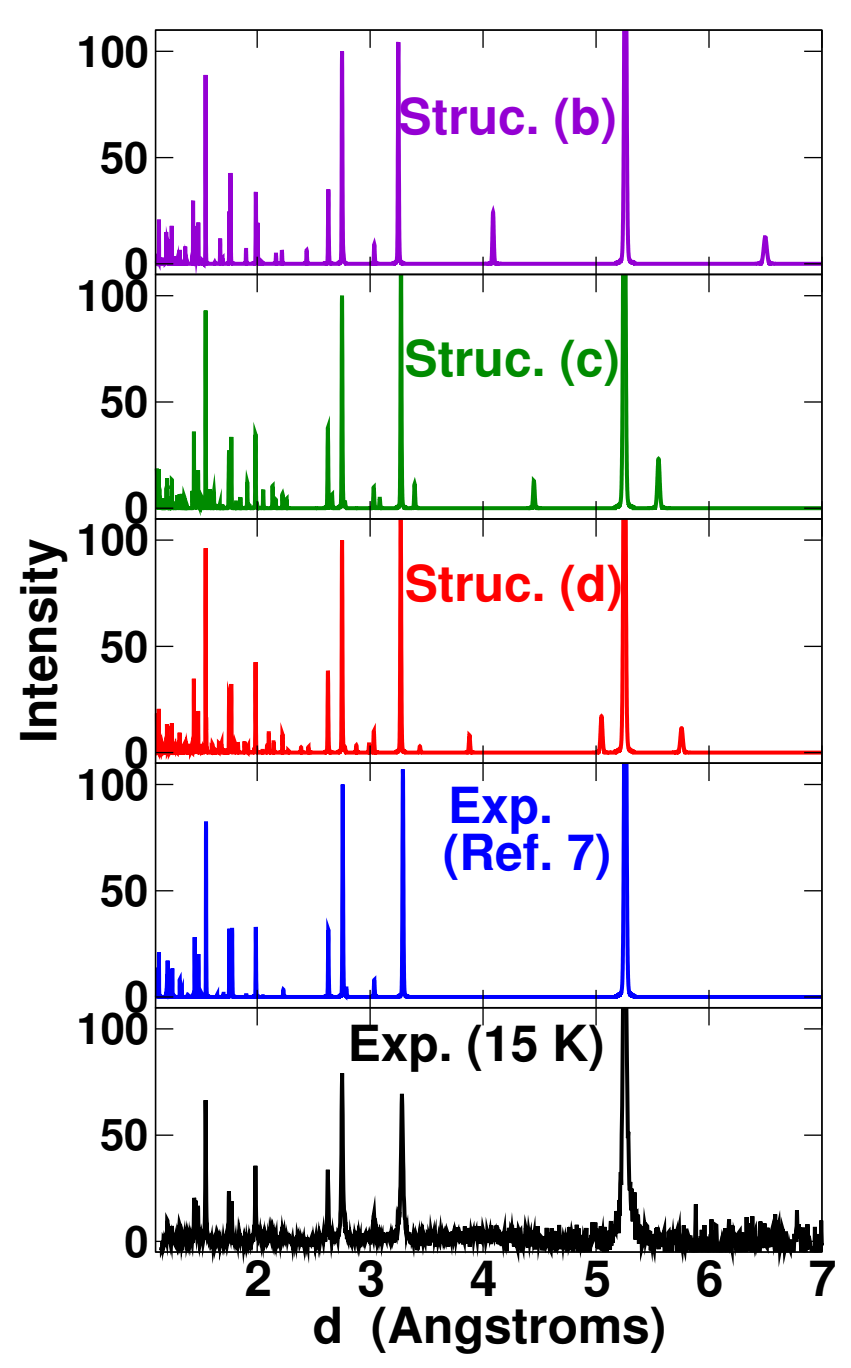

Figure 7: (Color online.) Simulated neutron diffraction patterns from the structural models shown in Fig. 3, compared with our low temperature neutron data and the room temperature structure by Mercier et al.[9] The intensity of the data and simulations were normalized so that the highest peaks at approximately $d=5.25 \AA$ are fixed at the intensity of approximately 200.

perature pattern in this work is essentially identical to the results reported by Mercier et al. [9]

Figure 6 compares simulated X-ray diffraction patterns for each of the structural variations shown in Fig. 3 with the measured pattern at $15 \mathrm{~K}$. In order to easily compare with the neutron data, the scattering angle $\Theta$ has been converted to the normal distance between diffracting planes $d$ according to the Bragg condition $d=\lambda /(2 \sin \Theta)$, where the $\mathrm{X}$-ray wavelength is $\lambda=1.54056 \AA$. The simulated patterns for the ordered supercell structures of Fig. 3 reproduce the main diffraction peaks quite well, but differ from the experimental patterns and from each other with the appearance of additional small intensity diffraction peaks mostly apparent at large $d$. If we assume that the powder sample is composed of grains or domains of material each having one of the possible structures, the diffraction pattern should be composed of an incoherent 
superposition of diffraction patterns for each of the structures. It is apparent that the incoherent average of the simulated patterns will reduce the intensity of the extra peaks and preserve the main features of the experimental pattern. The Mercier crystal parameters[9] which describe the structure in terms of the $50 \%$ occupancy of the $\mathrm{P}$ sites generates the same powder diffraction pattern. However, rather than having a completely random occupation, as described in Fig. 2, the actual $\mathrm{P}$ site occupation is restricted to $\mathrm{P}_{\uparrow}$ and $\mathrm{P}_{\downarrow}$ configurations.

We can make a similar comparison with the neutron diffraction data shown in Fig. 7. As expected, the diffraction peak positions as a function of diffraction plane distances $d$ are identical in the X-ray and neutron results, but because of the different cross section relationships, the intensity profiles of Fig. 7 and Fig. 6 are quite different. The simulated patterns for Struct. (b), (c), and (d) again show extra peaks at large values of $d$. In the X-ray pattern, the largest diffraction peak occurs at $d=2.76 \AA$ which corresponds to 12 equivalent $(2 \overline{1} 1)$ reflections in the hexagonal unit cell while another strong diffraction peak occurs at $d=3.29 \stackrel{\circ}{A}$ corresponding to 2 equivalent (002) reflections. In the neutron pattern the largest diffraction peak occurs at $d=5.26 \AA$ corresponding to 6 equivalent (100) reflections in the hexagonal unit cell. Interestingly, in these figures, the peak for the largest $d$ value occurs at $d=6.50 \AA$ corresponding to a (001) reflection in Struc. (b). For all the other structures and the experimental results, the $c$-axis symmetry allows only multiples of (002) reflections.

The refinement of our X-ray and neutron data and simulations for the lattice parameters $a$ and $c$ and fractional coordinate parameters $z_{P}$ and $x_{S}$ are summarized in Table 1 in terms of the hexagonal $P 6_{3} / \mathrm{mcm}$ unit cell. The numerical results are consistent with the conclusions from analysis of the X-ray patterns of Fig. 5, namely that the structure is invariant with temperature.

Table 1: Summary of structural parameters for $\mathrm{Li}_{4} \mathrm{P}_{2} \mathrm{~S}_{6}$. Calculated results were averaged from supercell simulations and the lattice parameters were scaled by 1.02 in order to account for the systematic underestimation of lattice size by the LDA exchange-correlation functional.

\begin{tabular}{l|l|l|l|l}
\hline & $a(\AA)$ & $c(\AA)$ & $z_{P}$ & $x_{S}$ \\
\hline This work at 15 K (X-ray) & 6.051 & 6.548 & 0.172 & 0.324 \\
This work at 15 K (neutron) & 6.055 & 6.553 & 0.172 & 0.326 \\
This work at 300 K (X-ray) & 6.075 & 6.597 & 0.172 & 0.324 \\
This work at 300 K (neutron) & 6.075 & 6.595 & 0.173 & 0.326 \\
Ref. [9]; X-ray at 293 K & 6.070 & 6.577 & 0.1715 & 0.3237 \\
Calculated structure b & 6.07 & 6.50 & 0.18 & 0.33 \\
Calculated structure c & 6.06 & 6.54 & 0.17 & 0.33 \\
Calculated structure d & 6.06 & 6.54 & 0.17 & 0.33 \\
\hline
\end{tabular}

\subsection{Stability of $\mathrm{Li}_{4} \mathrm{P}_{2} \mathrm{~S}_{6}$}

The $\mathrm{Li}_{4} \mathrm{P}_{2} \mathrm{~S}_{6}$ crystal was found to be thermally stable in vacuum up to $950^{\circ} \mathrm{C}$. Figure 8 compares the thermal gravimetric analysis (TGA) in Ar and in air. In an atmosphere of Ar gas, there is a small amount of weight loss in the sample, presumably due to a small amount of impurities in the Ar source. By contrast, when placed in air, the sample is stable (losing a weight percent of $2 \%$ or less) up to $280^{\circ} \mathrm{C}$. At temperatures higher than $280^{\circ} \mathrm{C}, \mathrm{Li}_{4} \mathrm{P}_{2} \mathrm{~S}_{6}$ loses nearly $25 \%$ of its mass (Fig. 8). Looking at these results in more detail, X-ray diffraction data were taken on the high temperature products at $280^{\circ} \mathrm{C}$ and at $350^{\circ}$ $\mathrm{C}$ (Fig. 9). In the latter case, reaction products of $\mathrm{P}_{2} \mathrm{O}_{5}$, $\mathrm{Li}_{4} \mathrm{P}_{2} \mathrm{O}_{7}$, and $\mathrm{Li}_{2} \mathrm{SO}_{4}$ were identified from the X-ray data. The room temperature pattern of $\mathrm{Li}_{4} \mathrm{P}_{2} \mathrm{~S}_{6}$ differs slightly from that shown in Fig. 6 because of small structural changes due to air exposure. Overall, the results indicate that $\mathrm{Li}_{4} \mathrm{P}_{2} \mathrm{~S}_{6}$ is much more stable than other lithium thiophosphates. For example $\mathrm{Li}_{3} \mathrm{PS}_{4}$ decomposes at room temperature in air.

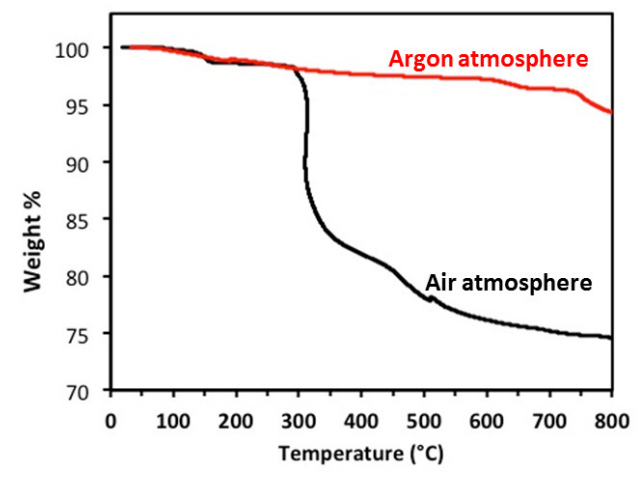

Figure 8: (Color online.) Thermal gravimetric analysis (TGA) of $\mathrm{Li}_{4} \mathrm{P}_{2} \mathrm{~S}_{6}$ showing the percentage weight remaining in the sample as a function of temperature when processed in Ar gas (red curve) or in air (black curve). $\mathrm{Li}_{4} \mathrm{P}_{2} \mathrm{~S}_{6}$ shows limited thermal stability in air until $280^{\circ} \mathrm{C}$.

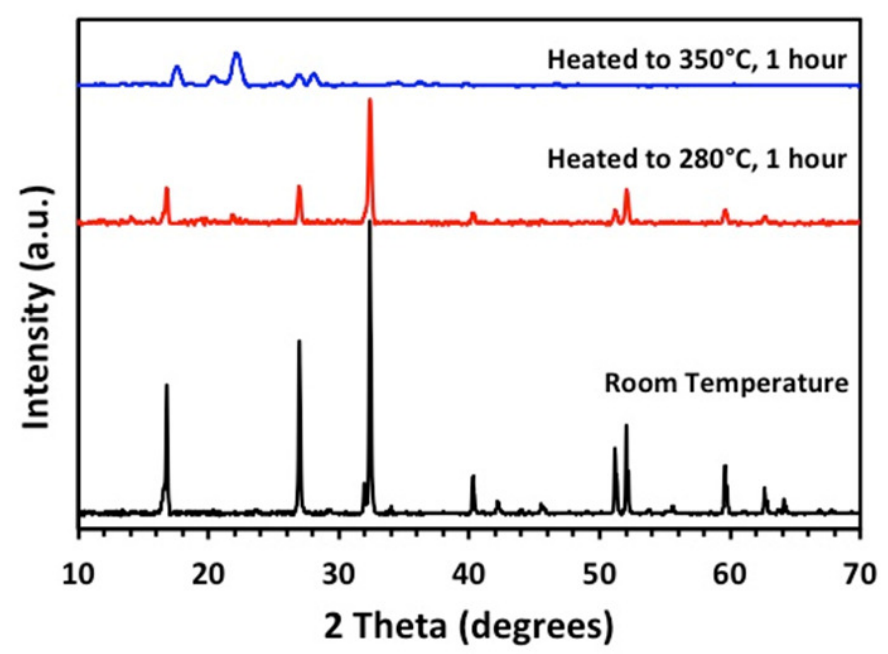

Figure 9: (Color online.) X-ray patterns of $\mathrm{Li}_{4} \mathrm{P}_{2} \mathrm{~S}_{6}$ processed at indicated temperatures in air. The $\mathrm{Li}_{4} \mathrm{P}_{2} \mathrm{~S}_{6}$ powder was heated/cooled at $100^{\circ} \mathrm{C}$ /hour to the indicated temperature and held at the temperature for 1 hour prior to the X-ray diffraction analysis. 


\subsection{Electrochemical measurements and simulations}

The samples in the form of pressed pellets prepared at $300 \mathrm{MPa}$ have a density of $2.14 \mathrm{~g} / \mathrm{cm}^{3}$. The ball-milled material allowed for the fabrication of denser pellets, which improved the ionic conductivity of $\mathrm{Li}_{4} \mathrm{P}_{2} \mathrm{~S}_{6}$. The pellets were pressed with $\mathrm{Al} / \mathrm{C}$ blocking electrodes to perform all impedance and Arrhenius measurements. The impedance measurements are given in the Appendix and the corresponding Arrhenius plot of the conductivity derived from the impedance measurements is shown in Fig. 10. The ionic conductivity of $\mathrm{Li}_{4} \mathrm{P}_{2} \mathrm{~S}_{6}$ is $2.38 \times 10^{-7} \mathrm{~S} \mathrm{~cm}^{-1}$ at $25^{\circ}$ $\mathrm{C}$ and $2.33 \times 10^{-6} \mathrm{~S} \mathrm{~cm}^{-1}$ at $100^{\circ} \mathrm{C}$. These results show that $\mathrm{Li}_{4} \mathrm{P}_{2} \mathrm{~S}_{6}$ has an activation energy (as defined by Eq. $5)$ of $0.29 \mathrm{eV}$. This activation energy is smaller than that measured for nano-porous $\beta-\mathrm{Li}_{3} \mathrm{PS}_{4}\left(E_{m} \approx 0.35 \mathrm{eV}\right)[5]$ but larger than the meta-stable superionic conducting material $\mathrm{Li}_{7} \mathrm{P}_{3} \mathrm{~S}_{11}\left(E_{m} \approx 0.12-0.18 \mathrm{eV}\right) .[32,33,7]$

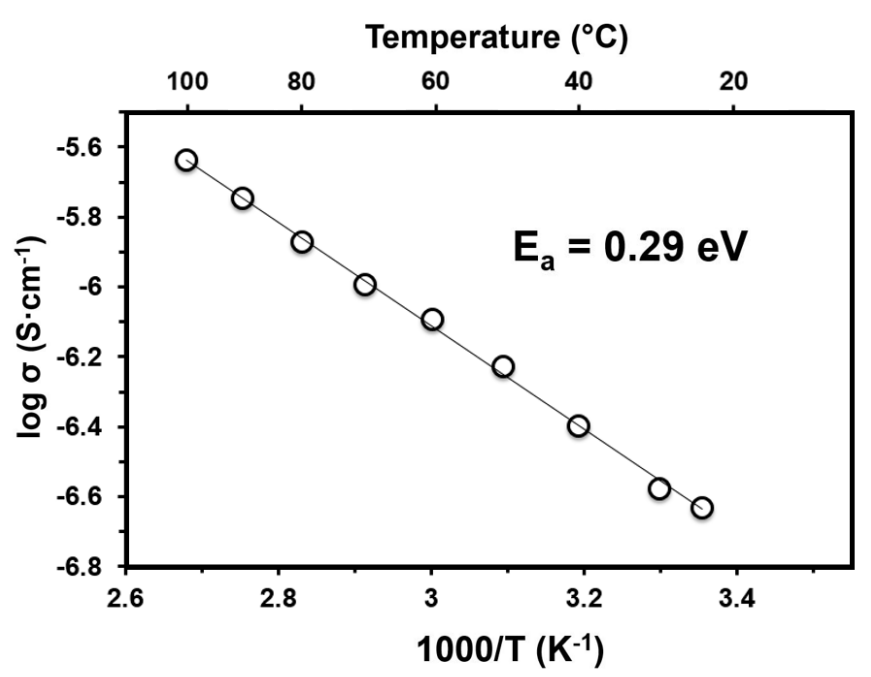

Figure 10: (Color online.) Arrhenius plot of the ionic conductivity (Eq. (5)) for $\mathrm{Li}_{4} \mathrm{P}_{2} \mathrm{~S}_{6}$.

In order to better understand the mechanism for ion conduction, we have carried out NEB calculations. First, we considered vacancy models based on 8 formula unit supercells of Struc. (d) shown in Fig. 3d. For this structure, unique $\mathrm{Li}$ vacancy hops can occur within the hexagonal plane between sites A, B, C, and D and perpendicular to the hexagonal plane between sites $\mathrm{C}, \mathrm{E}$, and $\mathrm{F}$ as shown in Fig. 11. The corresponding energy versus configuration diagrams are shown in Fig. 12, indicating that the vacancy migration energies are given by $E_{m}=0.6 \mathrm{eV}$ for paths both within a hexagonal plane and perpendicular to the plane. Using a supercell with a similar dimension, we also investigated the migration of vacancies in the $P \overline{3} 1 \mathrm{~m}$ structure shown in Fig. 3b, finding a minimum migration energy of $E_{m}=0.5 \mathrm{eV}$ for $\mathrm{Li}$ vacancies in a hexagonal layer between $\mathrm{P}_{2} \mathrm{~S}_{6}$ groups. The similarity of these two results suggest that for the vacancy mechanism, the effects of $\mathrm{P}_{\uparrow}$ and $\mathrm{P}_{\downarrow}$ disorder has little effect on $\mathrm{Li}$ ion migration barriers. However, by comparison of the calculated migration energies with the experimentally measured activation for $\mathrm{Li}_{4} \mathrm{P}_{2} \mathrm{~S}_{6}$, one can conclude that the $\mathrm{Li}$ ion conduction is unlikely to be explained by the vacancy hopping mechanism.

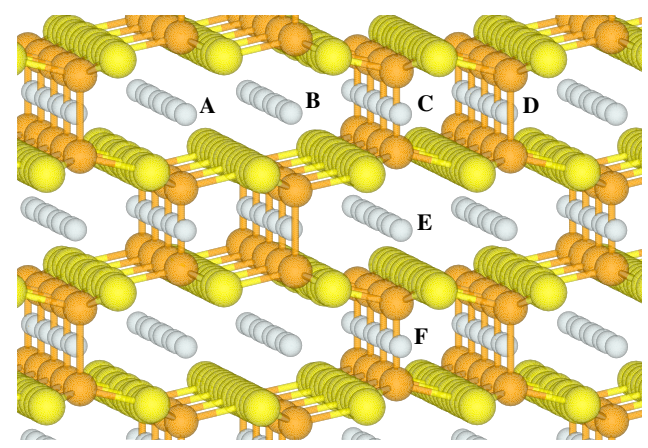

Figure 11: (Color online.) Ball-and-stick diagram of supercell of $\mathrm{Li}_{4} \mathrm{P}_{2} \mathrm{~S}_{6}$ in Struc. (d) shown in Fig. 3d, indicating vacancy positions A-F. The view point of this diagram is similar to that of Fig. 3d.
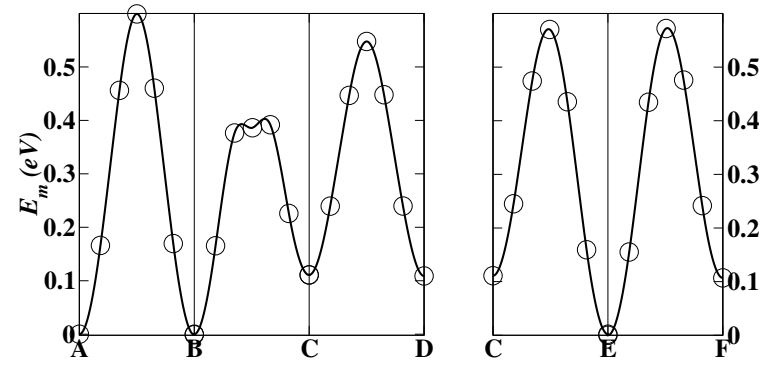

Figure 12: Energy path diagrams corresponding to the supercell model shown in Fig. 11 determined by NEB calculations of unique vacancy hops within the hexagonal plane (left graph) or along a $c$ axis (right graph). The zero of energy was taken as the lowest vacancy configuration.

We also considered possible interstitial Li ion migration mechanisms, focusing on the 8 formula unit supercells of Struc. (d) shown in Fig. 3d. In order to find possible meta-stable interstitial configurations, we relaxed a sizable number of initial configurations based on a coarse grid of possible interstitial sites with one fixed remote vacancy in order to keep the simulation cells with zero net charge. Several metastable interstitial sites were found, including the 4 neighboring sites shown in Fig. 13a. While these sites, which are roughly $2 \AA$ apart from each other and from nearby host lattice Li sites, do not span the supercell, they can give a reasonable approximation to a configuration diagram for a pure interstitial mechanism of Li ion migration as shown in Fig. 13b. For this partial path, the migration energy is $E_{m}=0.1 \mathrm{eV}$, suggesting that it is likely that the interstitial Li ion migration is energetically favorable for this material. More complicated processes, such as an interstialcy mechanism involving both interstitial and host lattice Li ions, could also be important for this system. As discussed in Sec. 2.2, the Arrhenius activation energy $E_{A}$ can also depend on the "formation" energy to 
produce an interstitial/vacancy pair, unless the sample has a native population of defects. Our simulations of possible interstitial/vacancy configurations result in an estimate of the formation energy to be more than $1 \mathrm{eV}$. Therefore, we conclude that our samples must have native populations of defects such $\mathrm{Li}^{+}$Frenkel interstitial/vacancy pairs.[34] (a)

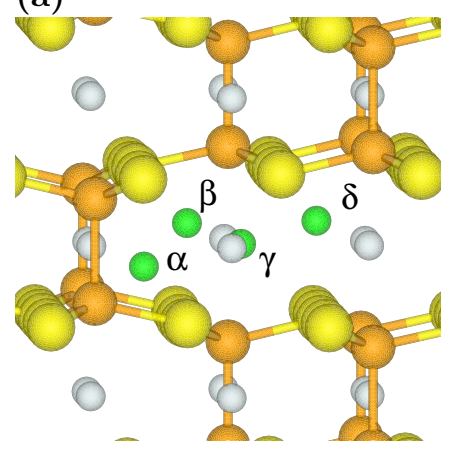

(b)

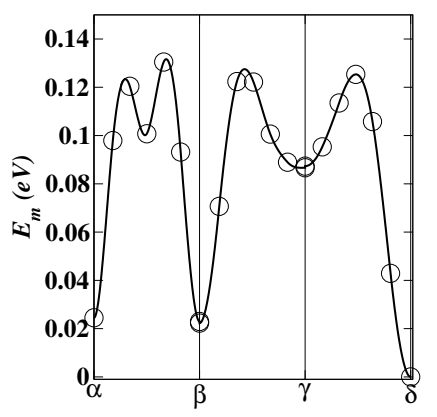

Figure 13: (Color online.) (a) Ball-and-stick diagram of metastable interstitial sites of the structure shown in Fig. 3d labeled $\alpha, \beta, \gamma$, and $\delta$ indicated in green and superposed on ideal lattice of Struc. (d). The view point of this diagram is similar to that of Fig. 3d and that of Fig. 11. (b) NEB energy path diagram for interstitial Li ion migration.

While we have shown that $\mathrm{Li}_{4} \mathrm{P}_{2} \mathrm{~S}_{6}$ is one of the more stable thiophosphate materials as shown in Fig. 8 it is interesting to ask the question of whether it is stable in the presence of a $\mathrm{Li}$ anode. When $\mathrm{Li}_{4} \mathrm{P}_{2} \mathrm{~S}_{6}$ is exposed to lithium metal, a noticeable reaction occurs between the solid electrolyte and the metal. While our experimental attempts to cycle a $\mathrm{Li} / \mathrm{Li}_{4} \mathrm{P}_{2} \mathrm{~S}_{6} / \mathrm{Li}$ cell have not yet been successful, ideal interfaces of the electrolyte with a $\mathrm{Li}$ anode give insight into the system.

For example, we considered one idealized cleavage of the crystal in a plane containing the hexagonal axis and containing complete $\mathrm{P}_{2} \mathrm{~S}_{6}$ units as shown in Fig. 14a. When this surface is exposed to an idealized Li metal interface and the system was allowed to relax, the system showed some decomposition as shown in Fig. 14b. In this case, $\mathrm{S}-\mathrm{P}$ bonds in the outer layer break in a similar way to that observed in the $\mathrm{Li}_{3} \mathrm{PS}_{4} / \mathrm{Li}$ system.[13] For $\mathrm{Li}_{4} \mathrm{P}_{2} \mathrm{~S}_{6} / \mathrm{Li}$ there seems to be a buffer layer of $\mathrm{Li}_{2} \mathrm{~S}$ formed at the interface, while the $\mathrm{P}-\mathrm{P}$ bonds remained intact. The partial density of states plot for this system is shown in Fig. 15.

Another example cleave was taken parallel to the hexagonal plane. In order to maintain the $\mathrm{P}_{2} \mathrm{~S}_{6}$ building blocks, this cleave results in a rough surface as shown in Fig. 16a. When Li metal layers are introduced into this supercell, the relaxed structure has broken $\mathrm{P}-\mathrm{S}$ bonds and $\mathrm{Li}_{2} \mathrm{~S}$ groups form. The optimized geometry is very sensitive to the details of the initial structure, an example of which is shown in Fig. 16b. In most of the cases studied the $\mathrm{P}-\mathrm{P}$ bonds remained intact. The partial density of states is shown in Fig. 17. (a)

(b)
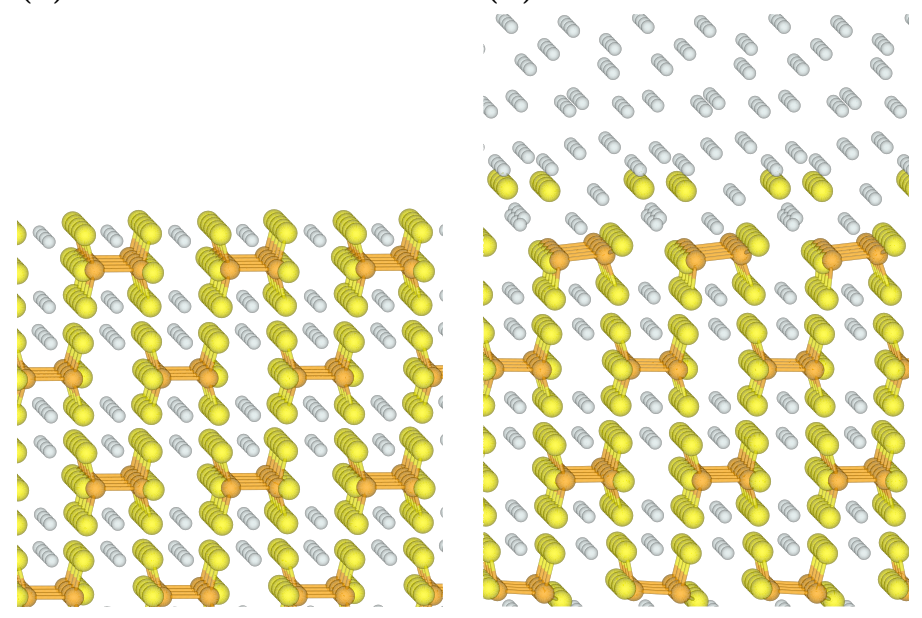

Figure 14: (Color online.) (a) Relaxed surface structure of $\mathrm{Li}_{4} \mathrm{P}_{2} \mathrm{~S}_{6}$ with Struc. (c) (Fig. 3c) cleaved perpendicular to the hexagonal plane with vacuum shown at the top of the diagram. (b) Relaxed structure of the surface in the presence of several layers of Li metal shown at the top of the diagram.

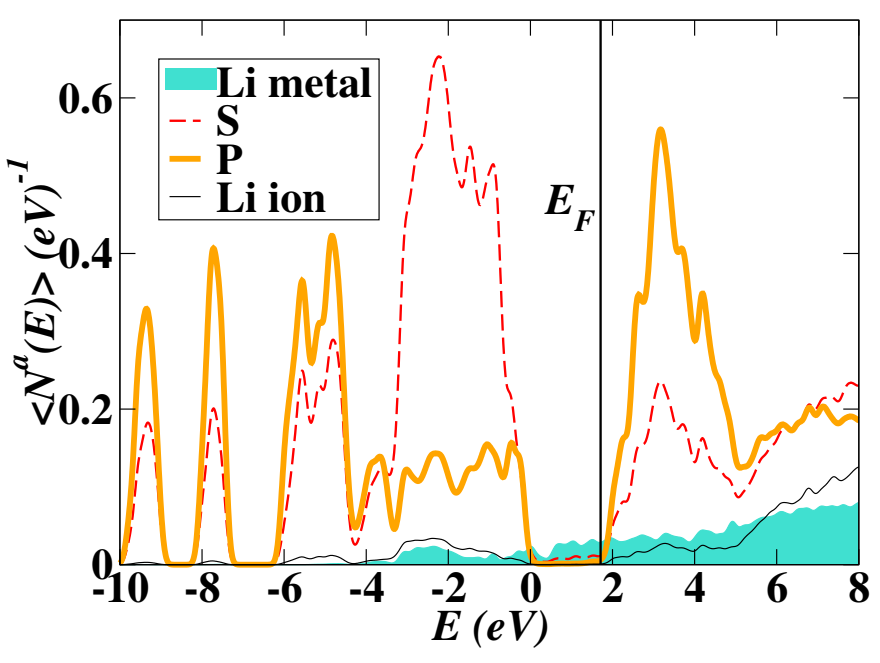

Figure 15: (Color online.) Partial density of states plot for the idealized interface shown in Fig. 14b. The zero of energy is adjusted to the bulk partial density of states plot of $\mathrm{Li}_{4} \mathrm{P}_{2} \mathrm{~S}_{6}$.

\section{Summary and Conclusions}

We have prepared and investigated pure samples of $\mathrm{Li}_{4} \mathrm{P}_{2} \mathrm{~S}_{6}$. In particular, we have shown that the disorder in the structure first identified by Mercier et al.[9] is due to energetic degeneracy in the placement of the $\mathrm{P}_{2} \mathrm{~S}_{6}$ building blocks of the structure. The simulation results are consistent with the X-ray and neutron diffraction measurements. We rationalize the observation that the structure is invariant with temperature by noting that within a given structure, transformation between the possible $\mathrm{P}-\mathrm{P}$ bond placements must have a high energy barrier. The relative stability of $\mathrm{Li}_{4} \mathrm{P}_{2} \mathrm{~S}_{6}$ compared with $\mathrm{Li}_{3} \mathrm{PS}_{4}$ correlates with its increased valence band width.

The activation energy determined from the Arrhenius 
(a)

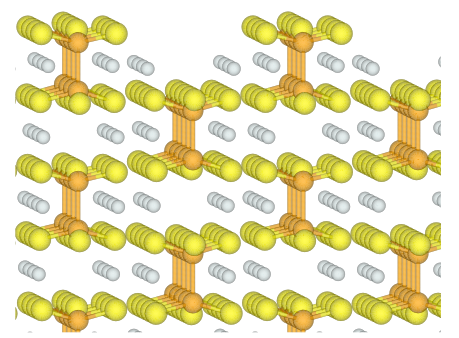

(b)

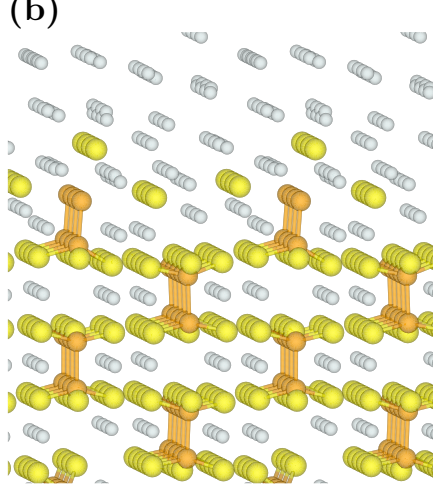

and shows prolonged stability in air when compared to other lithium thiophosphates. The results of this study provide insights towards better understanding of the family of lithium thiophosphate solid electrolytes as one of the very promising candidate materials for the implementation of safe high energy-dense batteries for large scale energy storage.

More generally, there are other members of the family of materials based on $\mathrm{P}_{2} \mathrm{~S}_{6}$ (hexathiohypodiphosphate) building blocks as recently shown by Kuhn et al.,[35] including $\mathrm{Na}_{4} \mathrm{P}_{2} \mathrm{~S}_{6}$, which may provide interesting comparisons to the present work and may be of interest to battery technology.

Figure 16: (Color online.) (a) Relaxed surface structure of $\mathrm{Li}_{4} \mathrm{P}_{2} \mathrm{~S}_{6}$ in Struc. (c) (Fig. 3c) cleaved parallel to the hexagonal plane with vacuum shown at the top of the diagram. (b) Relaxed structure of the surface in the presence of several layers of Li metal shown at the top of the diagram.

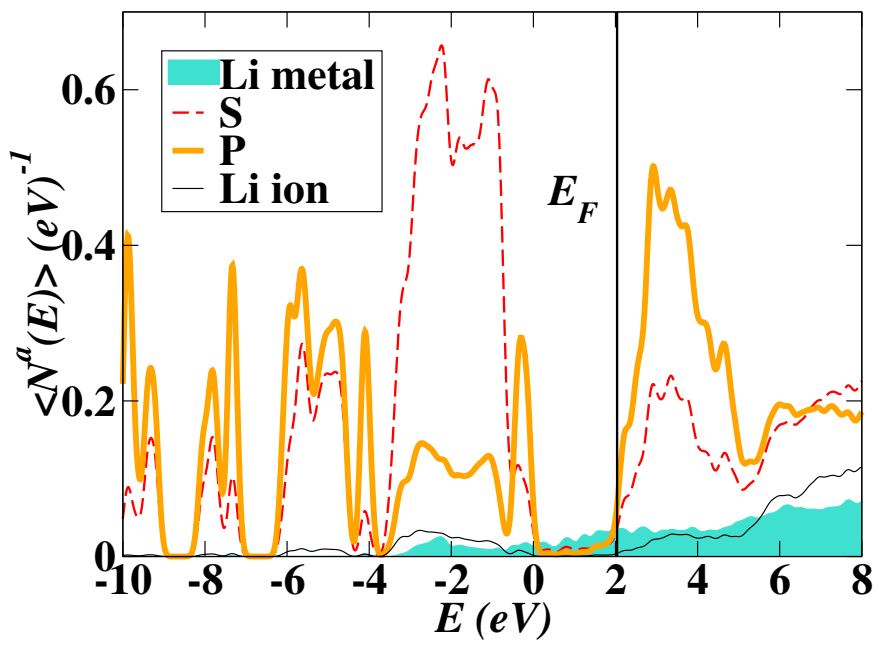

Figure 17: (Color online.) Partial density of states plot for the idealized interface shown in Fig. 16b. The zero of energy is adjusted to the bulk partial density of states plot of $\mathrm{Li}_{4} \mathrm{P}_{2} \mathrm{~S}_{6}$.

conductivity measurements is $E_{A}=0.29 \mathrm{eV}$, which is quite favorable. However for the samples we prepared, the magnitude of the ionic conductivity is very low. Simulations indicate that the mechanism for ionic conduction in this material is likely to involve interstitial processes. If the concentration of interstitial $\mathrm{Li}$ ions could be increased, perhaps the ionic conductivity could be improved. The impedance measurements were made using blocking electrodes. Cells prepared with pure $\mathrm{Li}$ electrodes could only be cycled a few times before shorting. Simulations on ideal surfaces suggest that the $\mathrm{Li}_{4} \mathrm{P}_{2} \mathrm{~S}_{6} / \mathrm{Li}$ interface can form a meta-stable buffer layer as does $\mathrm{Li}_{3} \mathrm{PS}_{4}$.[13] However, experiments show that for $\mathrm{Li}_{4} \mathrm{P}_{2} \mathrm{~S}_{6} / \mathrm{Li}$, this meta-stability is very fragile and typically not realized, in contrast to the $\mathrm{Li}_{3} \mathrm{PS}_{4} / \mathrm{Li}$ system. [5]

The current study has enhanced our understanding of the Li thiophosphate family of solid electrolytes, finding that $\mathrm{Li}_{4} \mathrm{P}_{2} \mathrm{~S}_{6}$ is formed at temperatures of $750^{\circ} \mathrm{C}$ or higher

\section{Acknowledgements}

The computational portion of this work was supported by NSF grant DMR-1105485. Computations were performed on the Wake Forest University DEAC cluster, a centrally managed resource with support provided in part by the University. Zachary D. Hood was supported by Higher Education Research Experiences (HERE) at Oak Ridge National Laboratory. The experimental portion of this work was primarily conducted at the Center for Nanophase Materials Sciences, which is a DOE Office of Science User Facility. Additionally, a portion of this research at ORNL's Spallation Neutron Source was sponsored by the Scientific User Facilities Division, Office of Basic Energy Sciences, US Department of Energy. We thank Jong Keum and Andrew Payzant for their assistance with cryogenic XRD measurements. Helpful discussions with Gayatri Sahu and Hui Wang from ORNL and William C. Kerr, Michael D. Gross, Keerthi Senevirathne, Cynthia Day, and Abdessadek Lachgar from WFU are gratefully acknowledged.

\section{Appendix}

A Nyquist or Cole-Cole plot of the real and imaginary parts of the impedance curves for $\operatorname{Li}_{4} \mathrm{P}_{2} \mathrm{~S}_{6}$ is shown in Fig. 18. The total ionic conductivity reported in Fig. 10 and detailed in Table 2 is determined by using the intercept of the semi-circle and the straight line as the resistance.

Table 2: Resistances and calculated ionic conductivities of $\mathrm{Li}_{4} \mathrm{P}_{2} \mathrm{~S}_{6}$ at different temperatures.

\begin{tabular}{ccc}
\hline Temperature $\left({ }^{\circ} \mathrm{C}\right)$ & $\mathrm{R}(\Omega)$ & $\sigma(\mathrm{S} / \mathrm{cm})$ \\
\hline 25 & 101030 & $2.38 \times 10^{-7}$ \\
30 & 88975 & $2.70 \times 10^{-7}$ \\
40 & 59143 & $4.06 \times 10^{-7}$ \\
50 & 40017 & $6.00 \times 10^{-7}$ \\
60 & 29326 & $8.18 \times 10^{-7}$ \\
70 & 23216 & $1.03 \times 10^{-6}$ \\
80 & 17537 & $1.37 \times 10^{-6}$ \\
90 & 13856 & $1.82 \times 10^{-6}$ \\
100 & 10283 & $2.33 \times 10^{-6}$ \\
\hline
\end{tabular}




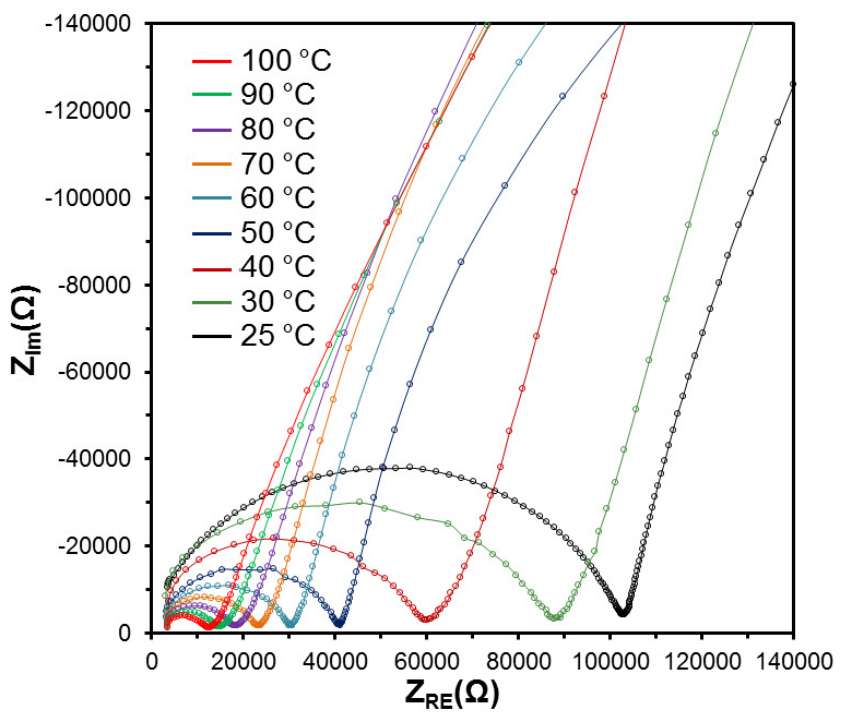

Figure 18: (Color online.) Impedance measurements for $\mathrm{Li}_{4} \mathrm{P}_{2} \mathrm{~S}_{6}$ at temperatures between $25^{\circ} \mathrm{C}$ and $100^{\circ} \mathrm{C}$ using blocking electrodes attached to the pellet having cross sectional area $1.27 \mathrm{~cm}^{2}$ and thickness $0.03 \mathrm{~cm}$. These impedance measurements are representative of results from other $\mathrm{Li}_{4} \mathrm{P}_{2} \mathrm{~S}_{6}$ pellets of similar size.

\section{References}

[1] Arthur L. Robinson and Jügen Janek. Solid-state batteries enter EV fray. MRS Bulletin, 39:1046-1047, 2014.

[2] Juchuan Li, Cheng Ma, Miaofang Chi, Chengdu Liang, and Nancy J. Dudney. Solid Electrolyte: the Key for High-Voltage Lithium Batteries. Advanced Energy Materials, 5:1401408, 2015.

[3] Yan Wang, William Davison Richards, Shyue Ping Ong, Lincoln J. Miara, Jae Chul Kim, Yifei Mo, and Gerbrand Ceder. Design principles for solid-state lithium superionic conductors. Nature Materials, 2015. URL http://www.nature.com/ doifinder/10.1038/nmat4369.

[4] Noriaki Kamaya, Kenji Homma, Yuichiro Yamakawa, Masaaki Hirayama, Ryoji Kanno, Masao Yonemura, Takashi Kamiyama, Yuki Kato, Shigenori Hama, Koji Kawamoto, and Akio Mitsui. A lithium superionic conductor. Nature Materials, 10(9):682686, 2011.

[5] Zengcai Liu, Wujun Fu, E. Andrew Payzant, Xiang Yu, Zili Wu, Nancy J. Dudney, Jim Kiggans, Kunlun Hong, Adam J. Rondinone, and Chengdu Liang. Anomalous high ionic conductivity of nanoporous $\beta-\mathrm{Li}_{3} \mathrm{PS}_{4}$. Journal of the American Chemical Society, 135(3):975-978, 2013.

[6] Keiichi Minami, Akitoshi Hayashi, and Masahiro Tatsumisago. Preparation and characterization of superionic conducting $\mathrm{Li}_{7} \mathrm{P}_{3} \mathrm{~S}_{11}$ crystal from glassy liquids. Journal of the Ceramic Society of Japan, 118:305-308, 2010.

[7] Akitoshi Hayashi, Keiichi Minami, and Masahiro Tatsumisago. Development of sulfide glass-ceramic electrolytes for allsolid-state lithium rechargeable batteries. J. Solid State Electrochem., 14:1761-1767, 2010.

[8] 2013. Independent private communications from Ezhiylmurugan Rangasamy and Zachary Hood.

[9] R. Mercier, J. P. Malugani, B. Fahys, J. Douglade, and G. Robert. Synthese, structure cristalline at analyse vibrationnelle de l'hexathiohypodiphosphate de lithium $\mathrm{Li}_{4} \mathrm{P}_{2} \mathrm{~S}_{6}$. Journal of Solid State Chemistry, 43:151-162, 1982.

[10] N. A. W. Holzwarth, N. D. Lepley, and Yaojun A. Du. Computer modeling of lithium phosphate and thiophosphate electrolyte materials. Journal of Power Sources, 196:6870-6876, 2011.
[11] Keiichi Minami, Fuminori Mizuno, Akitoshi Hayashi, and Masahiro Tatsumisago. Lithium ion conductivity of the $\mathrm{Li}_{2} \mathrm{~S}-$ $\mathrm{P}_{2} \mathrm{~S}_{5}$ glass-based electrolytes prepared by the melt quenching method. Solid State Ionics, 178(1112):837-841, 2007.

[12] A. C. Larson and R. B. Von Dreele. General Structure Analysis System GSAS, 2004. Los Alamos National Laboratory Report LAUR 86-748-748.

[13] N. D. Lepley, N. A. W. Holzwarth, and Yaojun A. Du. Structures, $\mathrm{Li}+$ mobilities, and interfacial properties of solid electrolytes $\mathrm{Li}_{3} \mathrm{PS}_{4}$ and $\mathrm{Li}_{3} \mathrm{PO}_{4}$ from first principles. Phys. Rev. $B, 88: 104103$ (11 pp), 2013.

[14] P. Hohenberg and W. Kohn. Inhomogeneous electron gas. Physical Review, 136:B864-B871, 1964.

[15] W. Kohn and L. J. Sham. Self-consistent equations including exchange and correlation effects. Physical Review, 140:A1133A1138, 1965.

[16] P. E. Blöchl. Projector augmented-wave method. Phys. Rev. B, 50:17953-17979, 1994.

[17] N. A. W. Holzwarth, A. R. Tackett, and G. E. Matthews. A Projector Augmented Wave (PAW) code for electronic structure calculations, Part I: atompaw for generating atom-centered functions. Computer Physics Communications, 135:329-347, 2001. Available from the website http://pwpaw.wfu.edu.

[18] X. Gonze, B. Amadon, P. M. Anglade, J. M. Beuken, F. Bottin, P. Boulanger, F. Bruneval, D. Caliste, R. Caracas, M. Cote, T. Deutsch, L. Genovese, Ph. Ghosez, M. Giantomassi, S. Goedecker, D. R. Hamann, P. Hermet, F. Jollet, G. Jomard, S. Leroux, M. Mancini, S. Mazevet, M. J. T. Oliveira, G. Onida, Y. Pouillon, T. Rangel, G. M. Rignanese, D. Sangalli, R. Shaltaf, M. Torrent, M. J. Verstraete, G. Zerah, and J. W. Zwanziger. Abinit: First-principles approach to material and nanosystem properties. Computer Physics Communications, 180(12):2582-2615, 2009. Code is available at the website http://www .abinit.org.

[19] Paolo Giannozzi, Stefano Baroni, Nicola Bonini, Matteo Calandra, Roberto Car, Carlo Cavazzoni, Davide Ceresoli, Guido L Chiarotti, Matteo Cococcioni, Ismaila Dabo, Andrea Dal Corso, Stefano de Gironcoli, Stefano Fabris, Guido Fratesi, Ralph Gebauer, Uwe Gerstmann, Christos Gougoussis, Anton Kokalj, Michele Lazzeri, Layla Martin-Samos, Nicola Marzari, Francesco Mauri, Riccardo Mazzarello, Stefano Paolini, Alfredo Pasquarello, Lorenzo Paulatto, Carlo Sbraccia, Sandro Scandolo, Gabriele Sclauzero, Ari P Seitsonen, Alexander Smogunov, Paolo Umari, and Renata M Wentzcovitch. Quantum espresso: a modular and open-source software project for quantum simulations of materials. J. Phys.: Condens. Matter, 21(39):394402 (19pp), 2009. Available from the website http://www.quantum-espresso.org.

[20] John P. Perdew and Yue Wang. Accurate and simple analytic representation of the electron-gas correlation energy. Phys. Rev. B, 45:13244-13249, 1992.

[21] N. A. W. Holzwarth, G. E. Matthews, R. B. Dunning, A. R. Tackett, and Y. Zeng. Comparison of the projector augmented wave, pseudopotential, and linearized augmented plane wave formalisms for density functional calculations of solids. Phys. Rev. B, 55:2005-2017, 1997.

[22] H. Jónsson, G. Mills, and K. W. Jacobsen. Nudged elastic band method for finding minimum energy paths of transitions. In B. J. Berne, G. Ciccotti, and D. F. Coker, editors, Classical and Quantum Dynamics in Condensed Phase Simulations, pages 385-404. World Scientific, Singapore, 1998.

[23] Graeme Henkelman, Blas P. Uberuaga, and Hannes Jónsson. A climbing image nudged elastic band method for finding saddle points and minimum energy paths. J. Chem. Phys., 113:99019904, 2000.

[24] Graeme Henkelman and Hannes Jónsson. Improved tangent estimate in the nudged elastic band method for finding minimum energy paths and saddle points. J. Chem. Phys., 113:9978-9985, 2000.

[25] Ryogo Kubo. Statistical-mechanical theory of irreversible processes. i. general theory and simple application to magnetic and 
conduction problems. Journal of the Physical Society of Japan, 12:570-586, 1957.

[26] Anton Kokalj. XCrySDen- an new program for displaying crystalline structures and densities. Journal of Molecular Graphics and Modelling, 17:176-179, 1999. Code available at the website http://www.xcrysden.org.

[27] Anton Kokalj. Computer graphics and graphical user interfaces as tools in simulations of matter at the atomic scale. Computational Materials Science, 28:155-168, 2003.

[28] Koichi Momma and Fujio Izumi. Vesta 3 for three-dimensional visualization of crystal, volumetric, an morphology data. Applied Crystallography, 44:1272-1276, 2011. Code available from the website http://jp-minerals.org/vesta/en/.

[29] H. T. Stokes and D. M. Hatch. Findsym: Program for identifying the space group symmetry of a crystal. J. Appl. Cryst., 38: 237-238, 2008. Available from the webpage http://iso.byu. edu/iso/isotropy.php.

[30] Mercury 3.5.1, 2014. Developed and distributed by the Cambridge Crystallographic Data Centre http://www.ccdc.cam.ac . uk/mercury/.

[31] Th. Hahn, editor. International Tables for Crystallography, Volume A: Space-group symmetry, Fifth revised edition. Kluwer, 2002. ISBN 0-7923-6590-9.

[32] Fuminori Mizuno, Akitoshi Hayashi, Kiyoharu Tadanaga, and Masahiro Tatsumisago. High lithium ion conducting glassceramics in the system $\mathrm{Li}_{2} \mathrm{~S}_{-} \mathrm{P}_{2} \mathrm{~S}_{5}$. Solid State Ionics, 177:2721$2725,2006$.

[33] Masahiro Tatsumisago and Akitoshi Hayashi. Preparation of lithium ion conducting glasses and glass-ceramics for all-solidstate batteries. Journal of Non-Crystalline Solids, 354:14111417,2008

[34] W. Hayes and A. M Stoneham. Defects and defect processes in nonmetallic solids. John Wiley \& Sons, 1985.

[35] Alexander Kuhn, Roland Eger, Jürgen Nuss, and Bettina V. Lotsch. Synthesis and structural characterization of the alkali thiophosphates $\mathrm{Na}_{2} \mathrm{P}_{2} \mathrm{~S}_{6}, \mathrm{Na}_{4} \mathrm{P}_{2} \mathrm{~S}_{6}, \mathrm{~K}_{4} \mathrm{P}_{2} \mathrm{~S}_{6}$, and $\mathrm{Rb}_{4} \mathrm{P}_{2} \mathrm{~S}_{6}$. Zeitschrift für anorganische und allgemeine Chemie, 640(5): 689-692, 2014. 